Théologiques

Théologiques

\title{
Dieu et ses mutants discursifs
}

\section{Walter Moser}

Volume 6, numéro 2, octobre 1998

Dieu interdit

URI : https://id.erudit.org/iderudit/024961ar

DOI : https://doi.org/10.7202/024961ar

Aller au sommaire du numéro

\section{Éditeur(s)}

Faculté de théologie de l'Université de Montréal

\section{ISSN}

1188-7109 (imprimé)

1492-1413 (numérique)

Découvrir la revue

\section{Citer cet article}

Moser, W. (1998). Dieu et ses mutants discursifs. Théologiques, 6(2), 33-49. https://doi.org/10.7202/024961ar

\section{Résumé de l'article}

Dans l'Occident chrétien, Dieu a occupé pendant longtemps la place première et centrale du système des discours. Aussi, la mort de Dieu, ou son interdiction, n'entraîne-t-elle pas la disparition de cette instance discursive, mais un " trou-en-forme-de-Dieu ». Ce texte propose l'analyse de quelques-uns des mutants discursifs de Dieu qui, lors d'un long processus de sécularisation, sont venus occuper la place vide de Dieu. D'une manière peu systématique, on observe quelques-unes de ces transformations discursives dans les domaines cognitif, pédagogique, esthétique, mais surtout politique. 
Théologiques 6/2 (1998) 33-49.

\title{
Dieu et ses mutants discursifs
}

\author{
Walter MOSER \\ Département de littérature comparée \\ Université de Montréal
}

Dans ce dossier de Théologiques nous nous demandons où est Dieu aujourd'hui, ou, plus prudemment, où en est la question de Dieu aujourd'hui. Ou encore : nous faisons le constat que, malgré le décret de la mort de Dieu, malgré la sécularisation avancée de notre monde et société, la figure et la question de Dieu sont loin d'être liquidées ou réglées. Dieu rôde encore. Revenant réfugié dans une crypte inaccessible aux assauts de la rationalité moderne, champion et héros secret de la résistance contre un monde abandonné à sa mécanique rationnelle, ultime espoir d'un monde désenchanté jusqu'aux os et qui tombe en proie à la pensée cynique, divinités-rejetons plurielles sortant du monumental corps abattu de la Divinité monothéiste, il semble maintenir présence et efficacité. Mais laquelle?

Dans la réflexion qui suit, j'aborderai la question par la voie de sa concrétisation discursive et me pencherai donc sur l'évolution de quelques mises en discours de l'instance "Dieu» ou de ce qui en a pris la place. Adoptant de plain-pied une approche discursive, je voudrais éviter de m'improviser théologien, sociologue, anthropologue. Voici l'hypothèse que je me propose d'examiner: Un système discursif qui a longtemps accordé à Dieu la place première, centrale et suprême, ne saurait, du jour au lendemain, se passer de cette figure; elle lui manquerait. En d'autre termes, la mort de Dieu laissera, dans un tel système, une insoutenable place vide. Le Dieu absent, chassé, éloigné laissera une marque indélébile, se sera transformé en une espèce de figuration en anamorphose repérable seulement pour qui se place dans la bonne perspective pour la reconnaître. Dans une ana logie photographique on pourrait dire que nous avons besoin d'un procédé particulier pour révéler la marque négative qu'aura laissée cette disparition sur la plaque sensible du discours. Mais la place 
centrale sera vide, ce qui ne manquera pas d'activer une loi de horror vacui discursif selon laquelle cette béance attirera une foule de figu rations secondaires qui seront appelées à remplir cette place vide. Le système se comportera selon la logique bien captée par cette formule qu'on trouve dans une des chansons du groupe U2: "Lookin' for to fill that GOD shaped hole ${ }^{1}$ ».

Cette formulation peut m'aider à préciser la tâche que je me propose ici : je voudrais, dans quelques-uns des discours qui circulent, faire apparaître ce trou-en-forme-de-Dieu et montrer comment on essaie sans cesse de le boucher. Comment cette vacance a favorisé le développement d'une multitude d'instances et de figurants qui parta. gent deux caractéristiques : ils ont un statut de secondarité souvent non-avoué (ce sont des ersatz, des vicaires, des lieu-tenants, des fairevaloir), et ce statut se trahit justement par leur aspiration à l'absolu et à une forme quelconque de transcendance.

Avant d'entamer cet examen, deux remarques préliminaires me paraissent nécessaires :

1. Il est évident que, dans l'espace qui m'est imparti ici, je ne saurais retracer toute l'histoire des mises en discours de l'instance «Dieu » et de ses multiples descendants et usurpateurs. Force m'est de renoncer d'emblée à la rigueur de l'analyse discursive et à la complétude qu'une telle étude devrait avoir l'ambition d'atteindre. Je ne propose donc qu'une traversée fragmentaire et ponctuelle du champ d'exploration ainsi circonscrit. Aussi opterai-je pour un style de type essayiste apte à prospecter ce champ, à suggérer plutôt que de démontrer, à sonder ici et là plutôt que de faire un relevé systéma tique, à ouvrir des liens d'interaction plutôt que de conclure.

2. À l'arrière-plan de cet essai, il y a la thèse de la modernité vue comme l'histoire d'un vaste processus de sécularisation. Ce que certains ont appelé et continuent d'appeler "la mort de Dieu " n'est pas survenu subitement, en quelque sorte par décret nietzschéen. Il s'agit plutôt d'un processus de transformation lent et imperceptible, entre coupé pourtant de poussées plus nettes. Une longue et lente séculari-

1 Je remercie Jean-Guy Nadeau qui m'a rendu attentif à cette chanson. 
sation $^{2}$ a eu lieu au cours de laquelle le sujet humain, collectif et individuel, s'est émancipé de la tutelle religieuse pour assumer luimême, en sujet autonome et responsable, l'agencement ${ }^{3}$ des processus historiques. Les humains ne sont plus les figurants et opérateurs terrestres des grands desseins divins, mais jouent leur propre histoire, conçue, exécutée et continuellement réinterprétée par eux-mêmes. $\mathrm{Ce}$ mouvement de longue durée se présente cependant sous un double jour, il a un envers et un endroit. Le versant positif et orgueilleux est celui de l'épopée moderne de l'émancipation qui raconte la prise en charge de son destin par l'espèce humaine, sa grande marche vers l'utopie optimiste. Le versant négatif peut le mieux être capté par les notions de mélancolie et de déréliction : mélancolie par rapport à un horizon de transcendance totalisant, rompu mais pas oublié; déréliction dans l'immanence dégrisante, qui fait pendant à l'orgueil et se manifeste surtout au moment où les doutes sur l'utopie moderne nous laissent en proie aux blues de la modernité.

L'aspect de ce processus de sécularisation qui sera au premier plan dans cet essai est la logique de sa réalisation discursive. Sur ce plan, elle se met en place en suivant une stratégie bien efficace qui est fondée sur le geste de la réutilisation réappropriante. On pourrait aussi parler de sa ruse qui consiste à se réapproprier les instances et structures discursives originairement théologico-religieuses et à les traduire du sacré au profance. Pour reprendre cette belle formule de Judith Schlanger, malgré ses élans de rupture et de redépart, malgré sa valorisation de la nouveauté, la modernité "pense la bouche pleine $^{4}$ », c'est-à-dire qu'elle se construit avec les matériaux et sur les restes d'un univers théocentrique et théocratique dont elle prend la place. Ainsi, pour ne donner qu'un exemple central dans cette

2 Voici, à titre d'exemple, quelques titres tirés d'une longue liste d'ouvrages qui portent sur le processus de sécularisation: Steve BRUCE (éd.), Religion and Modernisation: Sociologists and Historians Debate the Secularization. Oxford, Clarendon Press, 1992; Hans BLUMENBERG, Sekularisierung und Selbstbehauptung. Frankfurt a.M., Suhrkamp, 1976; Olivier TSCHANNEN, Les théories de la sécularisation. Genève, Droz, 1992.

3 Je me servirai ici de cette traduction du terme anglais " agency ".

4 Voir Judith SCHLANGER, Penser la bouche pleine. Paris-La Haye, Mouton, 1975. 
logique de la reconversion, la dimension eschatologique du christianisme se coulera dans la dimension utopique de la modernité.

\section{Le discours théocentrique}

Il peut paraître osé de reconstruire le fonctionnement de l'instance "Dieu " dans un univers théocentrique, surtout si on n'est pas théologien. Aussi me contenterai-je d'évoquer le fonctionnement discursif de cette instance et me limiterai-je à quelques traits saillants.

Dieu est l'alpha et l'oméga, il fonctionne comme instance de discours ultime et première, il est archè et télos. Il est le Super-Sujet qui est à l'origine de toute action. À lui renvoie en dernière instance toute action humaine, comme à l'instance transcendante qui la supplante en la garantissant, la rendant possible et lui donnant sens.

\subsection{Que fait l'instance divine?}

Instance se situant toujours en amont des choses, elle les fonde. Elle est l'instance fondatrice par excellence et devient, de ce fait, non-contournable. Personne ne saurait voir dans le dos ni par-dessus les épaules de Dieu. Tout tient de lui et vient de lui, car il est le Sujet Créateur au sens le plus fort du terme. C'est à lui que revient l'acte de Création premier dont dérivent tous les gestes de création secondaires confiés à des instances humaines.

Une autre activité attribuable à Dieu en premier est celle de la connaissance. En lui convergent toutes les modalités de connaissance, de lui dérivent toutes les capacités habilitant des êtres inférieurs à accéder à la connaissance : perception, intelligence, intel lect, raison, entendement, etc.

\subsection{Quelle est l'instance divine?}

En conséquence, Dieu réunit en lui les prédicats les plus totalisants qui soient. Le définir c'est donner dans l'absolu, s'en tenir aux termes superlatifs. Ainsi, entre autres choses, Dieu est l'Un et le Tout, il est ubique, éternel, omniscient, tout-puissant.

Dans la hiérarchie des regards, c'est lui qui a le regard le plus englobant et le plus surplombant. Mais aussi le plus pénétrant, puisqu'il sait radiographier les âmes. Sa vision est celle du totum 
simul, de la synopsis. Ses perceptions visuelles sont celles du panopticum parfait : rien ne peut lui échapper. Il est l'CEil vivant qui voit tout et pénètre partout.

À quelles stratégies discursives peut-on avoir recours pour affirmer cette primauté absolue, cette supériorité totale? Une des stratégies consiste à affirmer les deux termes d'une opposition: Dieu est à la fois une chose (prédicat $A$ ) et son contraire (prédicat non-A). Il est son et silence, il est centre et circonférence du cercle. En termes tropologiques, le discours sur Dieu développe alors un penchant pour l'oxymore. Ou, comme c'est le cas dans le discours mystique, il a recours à l'unio oppositorum, selon laquelle on identifie Dieu comme le lieu où les oppositions se résorbent en unité. Il est alors le tiers lieu où règne une logique de l'inclusion totale qui s'énonce toutefois selon les modalités du paradoxe : Dieu est à la fois transcendant et immanent, absent et présent, médiat et immédiat, lointain et proche, Sujet et Objet.

Une autre stratégie encore est celle du discours apophatique qui est la voie de la prédication négative : puisqu'on ne saurait affirmer directement les qualités de Dieu, on les approche négativement: Dieu n'est pas ceci, ni cela. En niant ainsi tous les prédicats concrets qui seraient limitatifs, on laisse ouvert le plein potentiel de tous les champs sémantiques ${ }^{5}$. Ou encore, on affirme sa non-prédicabilité, c'est-à-dire sa capacité d'excéder toute possibilité d'être nommé, réprésenté, énoncé : Dieu est l'Innommable, l'Irreprésentable, l'Indicible.

\subsection{Les scénarios narratifs et dramatiques}

Une autre manière encore d'aborder la représentation de Dieu consiste à l'insérer dans des scénarios de type narratif ou dramatique. Dans ce cas son vis-à-vis, son interlocuteur, celui qui lui donne la réplique ou qui lui joue de mauvais tours, c'est évidemment l'être humain. Mais comme l'être humain est issu d'un acte de création divine, cette altérité se ramène génétiquement à une mêmeté.

5 Dans la même logique, le sujet humain qui s'engage sur la voie de l'union mystique avec Dieu, doit se dévêtir progressivement de ses qualités concrètes et atteindre l'état d'un sujet sans qualité. 
Ayant recours à l'outil conceptuel assez rudimentaire qu'est la structure actantielle telle que développée par la narratologie sémiotique, on peut jouer divers scénarios.

\subsection{Structure actantielle}

La structure actantielle distingue un certain nombre d'actants engagés dans un schéma stable de relations représentant des dynamiques d'action :

$\begin{array}{cccc}\text { destinateur } \quad-\quad \text { objet } & - & \text { destinataire } \\ & \mid & & \\ \text { adjuvant } & \text { sujet } & & \text { opposant }\end{array}$

Ce schéma comporte une double syntaxe narrative :

1. un actant " sujet " cherche à s'approprier un actant " objet", et ceci contre les interférences négatives d'un actant « opposant » et avec l'appui d'un actant " adjuvant »

2. Un actant « destinateur» veut transmettre un actant « objet» à un actant « destinataire " moyennant l'action d'un actant "sujet "

Par exemple: Dieu (destinateur) transmet la vertu théologale (objet) de la charité à l'espèce humaine (destinataire), ce qui peut être représenté comme l'action d'un héros humain (sujet) qui, contre les interventions ou les mauvais conseils d'un adversaire (opposant) mais fort de l'appui et des bons conseils d'un ami ou complice (adjuvant) conquiert cet objet précieux.

On pourrait multiplier les exemples et découvrirait alors, moyennant les ajustements génétiques ou expansifs nécessaires, que Dieu peut occuper toutes les positions de la structure. Tantôt destinataire ou destinateur (il peut se donner à lui-même une humanité obéissante!), tantôt sujet ou objet (il peut prendre la place du héros ou encore celle de l'objet précieux), il peut se trouver en position d'adjuvant ou d'opposant par des ajustements génétiques, puisqu'il peut se muer en son double, Satan l'ange déchu, ou encore en Jésus, son propre fils envoyé sur terre parmi les humains. D'autre part, par expansion, il est également le sujet humain, puisqu'il a créé 
l'homme à sa propre image. Et il est finalement aussi l'univers, autre réalité issue de sa propre puissance créatrice.

Combinant cette grammaire des actants avec l'asémantisme de certaines définitions et surtout avec le discours apophatique, on pourrait mettre la position prépondérante de Dieu en analogie avec celle du Joker dans un jeu de cartes. Il s'agit de la carte qui, indéterminée, peut prendre n'importe quelle place dans le jeu, mais à laquelle, en vertu des règles de jeu, on accorde un pouvoir illimité sur toutes les autres. Une autre figure analogique, encore plus iconoclaste, et néanmoins tout aussi pertinente, serait celle du zéro. Dieu est le zéro: parfait dans sa figuration circulaire, et puissant puisqu'il peut, comme facteur de multiplication, annuler toute valeur - ou encore, ajouté en position finale, décupler la valeur de n'importe quelle position donnée 6 .

Certes, cet aperçu sur certains aspects des discours sur Dieu qui ont circulé dans notre culture ne saurait prétendre être complet, ni même être rigoureusement établi. En particulier par rapport aux nuances et complexité des discours théologiques, il représente une vulgarisation grossière. Mais l'enjeu de son établissement consiste justement à faire apparaître quelques lignes de force de l'efficacité " at large » de cette configuration discursive.

\section{Divers stades de la sécularisation}

\subsection{Le XVIIIe siècle}

Lançons une première sonde au XVIII ${ }^{\mathrm{e}}$ siècle, moment privilégié où la sécularisation commence à s'accélérer pour se solder par une poussée abrupte et violente dans la Révolution française. On y observe un double mouvement: d'une part Dieu s'humanise, il descend de son piédestal et se glisse dans des rôles humains. D'autre part Dieu s'éloigne, devient une instance lointaine qui n'intervient plus dans le cours de l'univers créé par lui et désormais régi par les lois de la nature; l'homme vient progressivement occuper le terrain inoccupé et s'attribuer les privilèges de Dieu. Il se divinise.

6 Voir à ce sujet la thèse de Joyce GogGin, The Big Deal : Card Games in 20th Century Novels (Université de Montréal, 1997), en particulier le chapitre sur l'histoire du symbole mathématique « zéro». 


\subsection{Humaniser Dieu}

À l'éloignement de Dieu (nécessaire pour le maintien de ses prédicats transcendants) correspond, en contrepartie, un rapprochement de Dieu qui efface ses prédicats, ouvrant ainsi le chemin à une anthropomorphisation du divin ${ }^{7}$. Qu'on se rappelle à ce sujet la prégnance, au XVIII e siècle, des métaphores mécanistes qui présentaient l'univers comme une machine géante, comme une horloge parfaite et qui appelaient une figure divine dans le rôle du grand machiniste, de l'ingénieur ou encore de l'horloger.

Un autre rôle professionnel humain qui fut destiné à Dieu par Lessing dans son L'Éducation du genre humain de $1780^{8}$, est celui d'éducateur. Dieu serait le plus grand maître d'école de tous les temps, puisque, face à son élève - le genre humain - qu'il s'agit d'amener à la connaissance des vérités ultimes, il aurait fait preuve de qualités pédagogiques exceptionnelles. La première de ses qualités est son omniscience; il s'agit d'un maître d'école qui, au départ, sait tout. La seconde raison de son excellence réside dans sa méthode pédagogique. Dans ses manuels scolaires, les textes de la Révélation biblique, dont il est indirectement l'auteur, il aura su adapter la rhétorique qu'il utilise aux degrés de développement de son élève. Au genre humain encore enfant, il parlait, dans l'Ancien Testament, en images et en termes figurés. Au prochain stade d'évolution de son élève (Nouveau Testament), il pouvait déjà révéler les vérités de manière plus directe. Et Lessing prévoit un troisième Testament, encore à venir, où Dieu parlera en termes directs et transparents le langage de la Raison, puisque son élève, en phase finale de sa formation, sera capable de saisir les vérités sans détour rhétorique.

Lessing combine ainsi le discours des Lumières (émancipation, progrès des Lumières et du bonheur) avec celui de la religion chré-

7 Voir l'entrée «Déchristianisation, laïcisation" (signé par Jean QUÉNIART) du Dictionnaire européen des Lumières, sous la direction de Michel DELON, Paris, Presses Universitaires de France, 1997, pp. 308 . 311.

8 Gotthold Ephraim LESSING, Die Erziehung des Menschengeschlechts. Le texte est de 1780; traduction française "L'Éducation du genre humain " dans Pierre-Henri TAVOILlot (dir.), Le crépuscule des Lumières. Paris, Cerf, pp. 11-29. 
tienne. Il projette directement la révélation divine sur la grande épopée du perfectionnement de l'espèce humaine; dans cette logique de sécularisation il réserve à Dieu le rôle du grand pédagogue.

\subsection{Diviniser l'humain}

Pour illustrer le mouvement en sens inverse, prenons l'exemple de l'Encyclopédie de Diderot et de D'Alembert, et plus spécifiquement le Discours préliminiare. Dans ce texte, D'Alembert se penche sur les modalités de perception et de connaissance :

L'homme qui combine aisément les idées, ne diffère guère de celui qui les combine avec peine, que comme celui qui juge tout d'un coup d'un tableau en l'envisageant, diffère de celui qui a besoin pour l'apprécier qu'on lui en fasse observer successivement toutes les parties $(43)^{9}$.

Ici le sujet humain - mais pas n'importe lequel - s'approprie le prédicat divin du totum simul, c'est dire qu'il accède à un mode de perception et de connaissance qui embrasse la complexité d'un tout selon la modalité temporelle du simultané. Le grand esprit, le philo. sophe ou encore le génie humain, pour inscrire la problématique dans un éventail de discours qui va du philosophique à l'esthétique, acquiert - ou plutôt s'attribue - la faculté divine de ce mode de connaissance. Certes, cette faculté reste exceptionnelle parmi les humains, elle n'échoit qu'aux super-individus, mais elle n'en devient pas moins accessible à l'humain. Ou, vu par l'autre bout de la lorgnette, l'humain ne s'installe pas moins, de la sorte, dans un privilège en principe divin.

Voici encore un exemple :

Il n'en est pas de même de l'ordre encyclopédique de nos connaissances. Ce dernier consiste à les rassembler dans le plus petit espace possible, et à placer, pour ainsi dire, le philosophe audessus de ce vaste labyrinthe dans un point de vue fort élevé d'où il puisse apercevoir à la fois les sciences et les arts principaux; voir d'un coup d'œil les objets de ses spéculations, et les opérations 
qu'il peut faire sur ces objets; distinguer les branches générales des connaissances humaines ${ }^{10}$.

D'Alembert répond ici à la question clé de toute encyclopédie : comment faire pour connaître, et donc dans quelle modalité discursive représenter la totalité des connaissances humaines? De nouveau, il distingue deux modalités, celle du philosophe qui, jouissant d'un lieu d'observation infiniment élevé, pourrait embrasser d'un seul regard la totalité du labyrinthe des connaissances, tandis que le commun des mortels devra se déplacer dans le labyrinthe dans l'espoir d'accéder un jour à la totalité par le cumul de ses expériences. Comme par hasard, ces deux modalités correspondent, dans l'organisation de l'Encyclopédie, d'une part au Système et au Tableau synoptique (pour le philosophe), et d'autre part au Dictionnaire qui aligne (pour le commun des mortels) entrée après entrée dans un labyrinthe organisé alphabétiquement.

La même divinisation du sujet humain exceptionnel est confirmée dans le discours romanesque du XVIII e siècle. Dans La Nouvelle Héloïse de Rousseau, le personnage de Wolmar n'est pas seulement un dieu-philosophe, mais il incarne une conscience morale surhumaine, en plus d'être doué d'une omniscience qui finit par le faire accéder à des qualités autrefois réservées à Dieu. Il n'est donc pas suprenant que Wolmar soit désigné par une expression qui faisait partie autrefois des représentations de Dieu : l'œil vivant. Et « œil vivant », il l'est au sens propre du terme : il voit tout, sait tout grâce à un œil fureteur et il est l'intelligence active qui accède à toutes les connaissances. Définitivement, Wolmar appartient à la catégorie des « grands esprits".

Le discours des Lumières distinguait entre " les grands esprits » et «le vulgaire ». Les grands esprits jouissent de privilèges qui les font accéder à certains prédicats auparavant réservés à l'instance divine : ils sont installés en hauteur, bénéficient donc d'une perspective surplombante, ce qui leur donne accès à une vision synoptique du labyrinthe où sont enfermés la plupart des sujets humains, et ils sont doués de capacités perceptives et cognitives qui leur épargne l'hu-

10 Jean LE ROND D'ALEMBERT, op.cit., p. 59. Italiques de mise en relief par W.M. 
maine lenteur de la saisie successive des parties d'un tout et les ins. talle d'emblée dans la jouissance du totum simul.

Cette élévation à la fois spatiale et cognitive, et même morale, des humains, du moins de certains humains, jusqu'à une puissance et un niveau divins s'accompagne, en contrepartie, d'une descente de la figure divine sur terre, son adoption d'une position humaine. Cette humanisation du Dieu chrétien connaît bien des variantes au XVIII ${ }^{e}$ siècle. Le modèle mécaniste de l'univers - le monde est une machine géante, une horloge bien conçue et bien huilée - appelle un concepteur et créateur dans le rôle humain du grand architecte, du machiniste ou de l'horloger. Horloger suprême et parfait, certes, mais néanmoins horloger, exerçant un métier bien humain et assez commun.

Il est vrai que l'observation de ces quelques exemples constitue à peine l'amorce d'une analyse des processus de sécularisation. Mais elle nous permet déjà de voir que ce processus est complexe et ne fonctionne pas à sens unique selon le modèle d'un énoncé du type "l'État se sépare de l'Église ". Nous avons plutôt découvert un mouvement de chassé-croisé entre les positions humaine et divine et leurs figurations respectives.

\subsection{Le XXe siècle}

Où en est ce processus aujourd'hui? Quelle distance avons-nous franchie depuis le siècle des Lumières? Depuis le décret nietzschéen de la mort de Dieu? Avant de proposer quelques éléments pour une réponse à cette question, précisons que je ne me propose pas de retracer historiquement tout le processus de sécularisation, mais une tâche bien plus restreinte qui consiste à observer, dans le contexte de ce processus, les procédés selon lesquels diverses instances discursives sont venues occuper la place vide laissée par la disparition de Dieu.

En jetant un regard rapide sur divers domaines où diverses instances et figures viennent occuper la place laissée vide de la sorte, commençons par relever quelques instances abstraites auxquelles certains discours ont tendance à accorder un statut semblable à celui dont jouit l'instance divine. Dans certains environnements discursifs, ces instances abstraites jouent en fait un rôle prépondérant et fonctionnent alors comme des agencements ou des actants nonanthropomorphes. 
Prenons, à titre d'exemple, les notions de pouvoir en politique, capital en économie et désir en psychanalyse. Dans chacune des disciplines en question, il existe certes, une manière très rigoureuse d'articuler ces instances. Mais il existe également, à divers degrés de vulgarisation, des utilisations de ces concepts qui n'obéissent pas aux mêmes exigences de rigueur, et qui ont tendance alors à les laisser choir dans la béance du trou-en-forme-de-Dieu. On leur accorde le statut d'ultime instance, avec préséance hiérarchique et priorité chronologique sur toutes les autres. Elles sont l'alpha et l'oméga de leur domaine, référence ultime et source première, on leur voue un culte quasi-religieux, sur le mode positif ou négatif, peu importe, tant que ce super-actant abstrait garde son pouvoir de figurer comme instance suprême dans toute chaîne narrative ou argumentative.

Plusieurs traits du discours sur Dieu sont ainsi mobilisés pour mettre en discours ces puissantes abstractions qui fonctionnent comme des divinitiés, chacune dans son secteur particulier. Ainsi donc, la résurgence de l'instance divine va de pair avec sa multipli cation sectorielle. On dirait que chaque domaine particulier cherche à proposer son concept fondateur pour avoir part à la grande opération qui consiste à trouver des substituts sécularisés pour Dieu ou, du moins, des concepts prenant la place de prédicats divins.

Dans la même veine, il y a également les figures anthropomorphes qui se glissent dans les niches vides de la Divinité. Depuis celle du Philosophe des Lumières que nous avons brièvement considérée ici, elles se sont multipliées au fur et à mesure que la place de Dieu s'est compartimentée selon la division du travail moderne. À chaque type d'activité humaine son aspiration divine, serait-on tenté de dire. En fait, on peut toujours repérer aujourd'hui, dans les secteurs les plus divers, la même tentation de " jouer à Dieu». Je ne saurais ici en parler que par prétérition, réservant la partie finale de cet essai à une analyse un peu plus poussée du domaine politique.

Il y a d'abord et toujours la figure du créateur artistique, qui a fait des efforts majeurs pour rompre avec un passé qui l'instaurait lieutenant de Dieu sur terre, à glorifier le Créateur divin par la répéti tion de son acte dans le domaine artistique. Mais, même les artistes les plus iconoclastes par rapport à cette lourde tradition ne sont pas 
au-dessus du soupçon de réintroduire dans leur figure et fonction des caractéristiques et prétentions divines ${ }^{11}$.

Étant donné le statut prestigieux des sciences dans notre société, il n'est pas surprenant de voir les scientifiques en première ligne, dans la course aux prédicats divins. D'autant plus qu'ils sont intrinsèquement reliés au développement des nouvelles technologies qui ont, de tout temps, alimenté l'image de l'humain démiurge. Aujourd'hui, c'est probablement la biologie qui favorise le plus le transfert de qualités divines au scientifique, puisque c'est le domaine où l'être humain est en train d'accéder aux secrets de la création divine (les codes génétiques), et s'apprête à les manipuler à sa guise.

Les pédagogues, également, sont sujets à l'aspiration vers les hauteurs par ce vent qui souffle par le trou-en-forme-de-Dieu. Ils doivent, chemin faisant, rencontrer le Dieu de Lessing qui est en train de descendre sur terre pour se mettre au niveau de son élève. $\grave{A}$ moins qu'ils ne suivent les "Instructions païennes" de Jean-François Lyotard qui propose de remplacer un modèle pédagogique de la verticalité (le professeur est installé sur les hauteurs de son savoir, l'élève dans la dépression de son ignorance et engagé dans un processus d'apprentissage qui a pour seul objectif de prendre la place du professeur) par un modèle narratif et dialogué ${ }^{12}$.

Il y a aussi l'homme politique à qui est confié, par délégation et par représentation, l'intérêt collectif; mais surtout la figure du politique qui détient le pouvoir : chef de gouvernement, président, premier ministre, mais aussi dictateur et tyran. Ces derniers, dont le XX siècle a produit plus que sa part, sont connus pour leur jalousie à l'égard de la toute-puissance divine, et surtout pour les moyens violents auxquels ils ont recours dans l'espoir d'y parvenir. Mais les leaders politques en régime démocratique ne sont pas non plus à l'abri de la tentation de " jouer à Dieu ".

11 Dans un exemple tout récent, cette prétention se trouve figurée dans le personnage du réalisateur de la Truman Show, dans le film du même nom, qui joue au Dieu ayant un pouvoir absolu sur le héros qu'il considère être sa "créature ".

12 Jean-François LYOTARD, Instructions päennes. Paris, Galilée, 1977. 
Pour finir, je m'attarderai plus longuement au domaine politique afin de faire une analyse un peu plus poussée de la manière dont l'État séculier organise le pouvoir et effectue l'exercice du pouvoir. À condition de prendre le terme "politique " dans un sens très large, nous avons certainement affaire à la réalité qui nous offre le champ d'observation et d'analyse le plus riche pour la problématique qui nous intéresse ici.

À commencer par la majuscule que la langue française accorde au mot «État ". Il s'agit là d'un traitement si rare d'un substantif en français qu'il est indice de quelque chose de particulier. Et, comme un des seuls autres mots jouissant du même traitement de faveur est "Dieu ", en se référant à une religion monothéïste, le lien est tout trouvé : du point de vue de la graphie «Dieu » et «État» se situent dans la même catégorie en français ${ }^{13}$.

Faisons donc l'hypothèse que, dans une situation de modernisation sécularisante avancée, l'État se glisse dans les souliers vides de Dieu. Le fait est aussitôt confirmé par l'émergence d'un concept et d'une organisation particulière de l'État appelée "État-providence ". Ici l'État s'approprie carrément un des prédicats divins qui consitutaient le noyau sémantique dur du concept de Dieu. Le transfert de ce prédicat de Dieu vers l'État représente un des exemples les plus évidents à l'appui de notre hypothèse générale : l'État-providence est le mutant le plus reconnaissable de Dieu dans notre monde sécularisé.

La providence est une gouverne prévoyante. Elle est attribuée à une instance capable de voir à tout, de pourvoir tout et surtout d'être en mesure d'assurer cette gouverne aussi dans l'avenir, c'est-à-dire de prévoir tout ${ }^{14}$. La divine providence est devenue proverbiale au point qu'il suffisait de mettre une majuscule à "providence" (la Providence) pour désigner Dieu par ellipse. L'État laïque a hérité

13 Certes, l'usage de la majuscule peut se justifier par la différentiation entre homonymes, mais la langue française, dans d'autres champs sémantiques, s'accommode bien de l'existence d'homonymes. D'ailleurs ce besoin de différentiation s'appliquerait alors aussi à la langue anglaise, ce qui est loin d'être le cas.

14 Étymologiquement le verbe "voir ", contenu dans "providence ", confirme le statut du sens de la vue comme le sens divin par excellence. 
dans ce cas d'un prédicat lourd de conséquences et surtout de responsabilités. Et l'enjeu est loin d'être purement conceptuel ou discursif. On observe parmi les fervents partisans de l'État-providence tout un comportement de culte et de déférence à l'égard de leur divinité laïque. Et des jérémiades face à la perspective de certaines restric tions imposées à la providentialité étatique.

À propos de restrictions : dans notre situation conjoncturelle très concrète, l'invocation du déficit zéro, et surtout des instances qui auront à décider de la cote de crédit de l'État, n'implique-t-elle pas, à son tour, la divinisation de certaines réalités purement politiques et économiques? Quand le déficit zéro devient l'ultime argument, absolu et non-questionnable, quand Moody's ou la Canadian Rating Bonds, ou encore d'autres agences d'évaluation de crédit, devient la haute instance qui, par son Jugement, décide du sort d'une collectivité humaine, ne la plaçons-nous pas en position divine?

De l'État-providence à l'État-surveillance il n'y a - hélas! qu'un pas. Un pas qu'on n'a pas le choix de ne pas faire. Les prédicats de Dieu viennent par paquets indissociables : providence ne va pas sans toute-puissance qui ne va pas sans omniscience. Et si l'État occupe le trou-en-forme-de-Dieu il aura à assumer tout le paquet de prédicats divins : il aura à jouer le rôle de Big Brother qui surveille tout, qui recueille et détient des informations sur tout et sur tout le monde.

Pour ce faire, pour se substituer à Dieu, aujourd'hui, l'État a recours aux technologies les plus avancées. La concrétisation sécularisée du discours sur Dieu emprunte la voie extra-discursive de la réalisation technologique. L'œil vivant et surplombant de Dieu plane partout au-dessus de nous sous la forme de caméras. Les caméras de surveillance sont omniprésentes dans notre vie; ouvertes en tout temps, et douées d'ubiquité, elles sont capables de capter le moindre de nos mouvements. Big Brother watches, dispersé en une multitudes d'yeux-caméras.

Quoi de plus divin que le grand nombre de satellites-espions avec leurs "yeux " surplombants, braqués sur tel ou tel endroit de la surface de la terre. Circulant dans le ciel, c'est l'œil de Dieu qui nous voit, nous observe, enregistre nos faits et gestes. Mais il ne s'agit pas seulement d'un œil bionique augmentant les capacités de l'organe humain; il s'agit de systèmes complexes, capables d'un regard fure- 
tant, pénétrant. En plus du regard optique, il y a le regard infrarouge, radiographique, echographique... tous ces systèmes de fabrication humaine fonctionnent de concert pour égaler l'ancienne pulsion scopique divine.

Mais Big Brother ne regarde pas seulement, il tient également registre de tout ce qu'il voit et sait. Tout s'inscrit dans le grand registre, sauf que la technologie a changé. Il ne s'agit plus de ce qui est écrit là-haut, du grand rouleau où la plume de Dieu inscrivait tout $^{15}$. On procède désormais à l'enregistrement électronique, à la collecte de donneés numérisées, bien plus rapide, fiable et puissante. Autrefois, ces données étaient dispersées, déposées dans différents ordinateurs appartenant à différents services gouvernementaux. Aujourd'hui le gouvernement, voulant jouer à Dieu pour étendre son contrôle, pense colliger toutes ces données et les intégrer en un réseau central. Décidément, la technologie est un excellent adjuvant pour permettre à l'État de jouer à Dieu.

À l'époque de Jacques le Fataliste et son maître ${ }^{16}$, il pouvait encore paraître rassurant de savoir que "tout était écrit là-haut ". Aujourd'hui, par contre, le perfectionnement rapide des performances gouvernementales en omniscience électronique fait peur. Combiné avec les autres systèmes de surveillance et de contrôle, il suscite de la paranoïa.

Information control is the contemporary version of God's eternal knowledge of each individual's ultimate damnation or salvation, and both theology and computer technologies naturally produce paranoid fears about how we are hooked into the system, about the connections it has in store for us 17 .

Et voilà le résultat de notre émancipation moderne qui devait nous libérer des instances transcendantes et nous donner le contrôle

15 C'est sans aucun doute le poète et graveur anglais William Blake, de la fin du XVIII $I^{e}$ siècle, qui nous a donné les représentations les plus marquantes de Dieu-le-scribe qui tient registre.

16 Roman de Denis DIDEROT, composé entre 1773 et 1775 et publié pour la première fois en 1796 .

17 Leo BERSANI, "Pynchon, Paranoia, and Literature", Representations 25 (1989) 99-118; p. 103. 
sur notre propre destin! Une espèce de dialectique de l'émancipation a imperceptiblement eu lieu et nous dépose dans une illiberté tout aussi grave que celle, au départ, qui nous voyait sujets de la puissance de Dieu. Assisté des technologies les plus modernes, l'État nous aura réduits à un assujettissement qui ressemble beaucoup à celui exercé par la toute-puissance divine. Sauf que l'État n'a plus les moyens de son ambition de jouer à la providence de sorte que, de ses prétensions divines, il risque de nous rester que la surveillance...

\section{Pour conclure}

Pour finir, un mot en faveur de la littérature. Comme bien des fois dans l'histoire occidentale, la littérature nous sert de early warning system. Elle nous lance un avertissement devant ce qui risque de nous arriver. Grâce à sa fictionalité elle peut faire des anticipations de ce qui nous attend. Du 1984 de George Orwell, écrit en 1949 pour un avenir encore fictionnel, l'anticipation appartient déjà au passé. Et la fiction est en fait déjà dépassée par la réalité. Mais dans les œuvres de Calvino, Delillo et Pynchon - pour ne mentionner que ces trois grands noms contemporains - nous trouvons aujourd'hui une thématisation assez insistante de la paranoïa qui s'installe quand l'État séculier s'apprête à vouloir trop fidèlement adopter les contours de Dieu après l'avoir expulsé des affaires d'État.

Cette rapide et cahotante calvalcade à travers quelques mutations modernes de l'instance discursive "Dieu " nous amène à la conclusion que, de Dieu, il n'y a que le nom avec majuscule qui soit interdit. Sa configuration discursive est encore bien en place, investie qu'elle est par de multiples figurations qui aspirent à remplir ce trou-en-forme-de-Dieu. L'efficacité de son fonctionnement discursif n'a pas faibli sous les menaces de sa sécularisation. Bien au contraire, elle a donné naissance à d'innombrables rejetons, les uns plus vigoureux que les autres.

Dans le domaine discursif, la configuration «Dieu " s'est avérée un véritable Protée, ce héros mythologique qui, maître de la métamorphose, reste toujours le même sous des apparences infiniment changeantes. Ou, pour le dire avec une figure qui fonctionne de la même manière, mais qui est tirée de la contemporanéité culturelle : "Dieu » est devenu un transformer, un héros mutant qui change d'allure, de nom, d'identité en un rien de temps. Un véritable Protée postmoderne qui rejoue les fantasmes les plus anciens. 